GA-A23949

\title{
DETAILED MEASUREMENTS OF THE ELECTRON CYCLOTRON CURRENT DRIVE EFFICIENCY ON DIIID
}

\author{
by \\ C.C. PETTY, R. PRATER, J. LOHR, T.C. LUCE, W.R. FOX, \\ R.W. HARVEY, J.E. KINSEY, L.L. LAO, and M.A. MAKOWSKI
}




\section{DISCLAIMER}

This report was prepared as an account of work sponsored by an agency of the United States Government. Neither the United States Government nor any agency thereof, nor any of their employees, makes any warranty, express or implied, or assumes any legal liability or responsibility for the accuracy, completeness, or usefulness of any information, apparatus, product, or process disclosed, or represents that its use would not infringe privately owned rights. Reference herein to any specific commercial product, process, or service by trade name, trademark, manufacturer, or otherwise, does not necessarily constitute or imply its endorsement, recommendation, or favoring by the United States Government or any agency thereof. The views and opinions of authors expressed herein do not necessarily state or reflect those of the United States Government or any agency thereof. 


\title{
DETAILED MEASUREMENTS OF THE ELECTRON CYCLOTRON CURRENT DRIVE EFFICIENCY ON DIIID
}

\author{
by \\ C.C. PETTY, R. PRATER, J. LOHR, T.C. LUCE, W.R. FOX, ${ }^{*}$ \\ R.W. HARVEY, $†$ J.E. KINSEY,‡ L.L. LAO, and M.A. MAKOWSKA
}

This is a preprint of a paper to be submitted to Nucl. Fusion.

*Princeton Plasma Physics Laboratory, Princeton, New Jersey.

${ }^{\dagger}$ CompX, Del Mar, California.

‡Lehigh University, Bethlehem, Pennsylvania.

†Lawrence Livermore National Laboratory, Livermore, California.

Work supported by

the U.S. Department of Energy under

Contract Nos. DE-AC03-99ER54463, DE-AC02-76CH03073, DE-FG03-99ER54141, DE-FG02-92ER54141, and W-7405-ENG-48 


\begin{abstract}
Electron cyclotron current drive (ECCD) experiments on the DIII-D tokamak are solidifying the physics basis for localized, off-axis current drive, the goal being to validate a predictive model for ECCD. The ECCD profiles are determined from the magnetic field pitch angles measured by motional Stark effect (MSE) polarimetry. The measured ECCD switches from the co to the counter direction as the toroidal injection angle is varied with a profile width that is in accordance with ray tracing calculations. Tests of electron trapping in low beta plasmas show that the ECCD efficiency decreases rapidly as the deposition is moved off-axis and towards the outboard side of the plasma, but the detrimental effects of electron trapping on the current drive are greatly reduced in high beta plasmas. Overall, the measured ECCD is in good agreement with theoretical calculations using a quasilinear Fokker-Planck code over a wide range of injection angles and plasma parameters.
\end{abstract}




\section{Introduction}

Electron cyclotron waves can efficiently drive localized non-inductive current in toroidal devices for a number of applications. For example, electron cyclotron current drive (ECCD) near the $q=3 / 2$ surface, where $q$ is the safety factor, has been used to stabilize the $(m, n)=(3,2)$ neoclassical tearing mode on several tokamaks $[1,2$, $3,4,5]$. Modification of the current profile using ECCD also has been demonstrated for reversal of the magnetic shear $[6,7,8]$ and sawtooth suppression $[9,10,11]$. More global applications for ECCD have been fully non-inductive current drive in tokamaks $[11,12,13,14]$ and bootstrap current compensation in stellarators [15]. The applications of ECCD are therefore both numerous and complex; unfortunately, the physics of ECCD is also complex since the interaction of electron cyclotron waves with electrons is very localized in both velocity space and real space. Thus, a validated theoretical model of ECCD is essential for its optimal use in fusion devices. Without this, the ECCD results from one device, or even one application, cannot readily be transferred to another device or application.

Electron cyclotron current drive results from the selective heating of electrons traveling in one toroidal direction to decrease their collision frequency, and thus increase their contribution to the toroidal current compared to their unheated counterparts moving in the opposite direction $[16,17]$. For off-axis current drive, this current drive mechanism is offset by the mirror trapping of electrons in toroidal geometry that drives current in the reverse direction [18]. The opposition between these two current drive mechanisms makes it imperative to study the influence of electron trapping on ECCD to determine the optimal regime for efficient generation of off-axis current. The theoretical ECCD can be written in the standard form [19]

$$
\frac{I_{e c}}{P_{e c}}=\frac{\epsilon_{0}^{2}}{e^{3}}\left(\frac{k T_{e}}{\ln \Lambda R n_{e}}\right) \zeta\left(Z, \epsilon, \theta_{p o l}, w\right),
$$

where $I_{e c}$ is the current driven by electron cyclotron waves, $P_{e c}$ is the absorbed wave power, $\epsilon_{0}$ is the permittivity of free space, $e$ is the charge of an electron, $k$ is the Boltzmann constant, $\ln \Lambda$ is the Coulomb logarithm, $R$ is the plasma major radius, and $T_{e}$ and $n_{e}$ are the electron temperature and density at the ECCD location. The theoretical complexity is contained in the dimensionless function $\zeta$, unique to each form of rf current drive, that depends upon the ion charge $Z$, inverse aspect ratio

$\epsilon$, poloidal location where the wave heating occurs $\theta_{\text {pol }}$, and wave phase velocity normalized to the electron thermal velocity $w$. In the low power density limit [20], the theoretical ECCD can be calculated from the relativistic, linearized Fokker-Planck equation using ray tracing codes $[21,22,23]$. However, a more complete Fokker- 
Planck treatment relevant to present day experiments requires that the effects of rf quasilinear diffusion and the dc parallel electric field be included. In this paper, the theoretical ECCD is calculated using the bounce-averaged, quasilinear Fokker-Planck code CQL3D [24], which is the most complete model of ECCD available to us.

Previous ECCD studies on tokamaks and stellarators have mainly measured the magnitude of the driven current from the change in the loop voltage at the plasma surface required to maintain a constant plasma current $[25,26]$. On the T-10 tokamak, the experimental ECCD was in qualitative agreement with quasilinear Fokker-Planck calculations, but the interpretation was complicated by sawteeth, transport losses, and non-optimum launch polarization so quantitative agreement was not obtained $[27,28,29]$. The effect of the residual dc parallel electric field on ECCD predicted by Fokker-Planck calculations was confirmed in the COMPASS-D [30] and DIII-D $[31,32]$ tokamaks. This dc electric field effect also may have been responsible for the missing counter ECCD on the RTP tokamak [33, 34]. In addition, many tokamak experiments have reported that the ECCD efficiency decreases as the power deposition location is moved away from the plasma center either by varying the magnetic field strength $[27,28,29,33,34]$ or by changing the poloidal steering of the ECCD launcher $[11,14,35]$. On the W7-AS stellarator, the role of trapped electrons in reducing the ECCD efficiency was clearly demonstrated in several experiments that utilized W7AS's flexibility to operate at different magnetic field configurations [25]. Additionally on W7-AS, co and counter ECCD were found to agree with linear theory at low power densities, whereas at high power density the ECCD was less than the linear theory especially for co injection [36, 37]; this may have been due to non-linear effects but Fokker-Planck calculations including stellarator specific loss-cone effects could not explain the measured degradation of the ECCD efficiency at extremely high power density [38].

The first localized measurements of the ECCD profile were made on the DIII-D tokamak using motional Stark effect (MSE) polarimetry [39, 40]. These experiments measured not only the ECCD magnitude but also the radial location and profile width as well. The analysis technique was later refined which resolved discrepancies between the measured and theoretical profile width and the dependence of the ECCD efficiency with increasing trapped electron fraction [35, 41]. Localized measurements of the ECCD profile using MSE data also has been made on the JT-60U tokamak, where the measured current drive was found to be in agreement with linear theory $[42,43]$.

The goal of the experiments reported in this paper is to experimentally validate the ECCD efficiency calculated by a quasilinear Fokker-Planck model, including the effect 
of the residual parallel electric field. In particular, the effect of electron trapping on the ECCD efficiency will be examined. Using internal magnetic measurements from the MSE diagnostic, localized driven currents as small as 1\% of the total plasma current can be measured accurately. As a result, the physics of ECCD can be explored in unprecedented detail since the ECCD efficiency can be determined over a wide range of conditions. These ECCD experiments satisfy all of the theoretical underpinnings, such as full absorption of the wave energy before the cold plasma resonance is reached and good confinement of the heated electrons. The ECCD direction is scanned from the co to the counter direction by changing the toroidal injection angle to vary the parallel index of refraction of the electron cyclotron waves in the plasma. This allows the trapping effects for co and counter ECCD to be studied separately in this paper. The effect of trapped electrons is varied by changing the location of the electron cyclotron resonance in real space to affect the magnetic geometry, as well as changing the resonance location in velocity space relative to the trapping-passing boundary by varying the electron beta.

The organization of this paper is as follows: in Section 2, the DIII-D tokamak, diagnostics, and ECCD system are described. The ECCD analysis method is discussed in Section 3 and typical ECCD profiles are shown. The dependences of the ECCD efficiency on the electron beta, parallel index of refraction, and the poloidal and radial locations are shown in Section 4, and the experimental results are compared with quasilinear Fokker-Planck calculations. The conclusions of this paper are presented in Section 5. 


\section{Experimental Setup}

These ECCD experiments are done on the DIII-D tokamak [44], typical parameters for which are major radius $R=1.7 \mathrm{~m}$, minor radius $a=0.6 \mathrm{~m}$, elongation $\kappa=1.8$, toroidal magnetic field strength $B_{T}=1.65-2.15 \mathrm{~T}$, and plasma current $I_{p}=0.6-$ 1.3 MA. The plasma is fueled by deuterium gas puffing and deuterium neutral beam injection (NBI), and the vessel walls are boronized to reduce the impurity influx during auxiliary heating. These experiments use both L-mode and H-mode plasmas, usually with a double null divertor plasma shape, although some L-mode plasmas use an inside-wall limiter shape to suppress the L-H transition while operating at relatively high beta.

Up to four gyrotron oscillators operating at $110 \mathrm{GHz}$ are used in these experiments, with a maximum combined power of $2.1 \mathrm{MW}$ injected into DIII-D [45, 46, 47]. After the non-Gaussian output from the gyrotron is converted to a Gaussian beam suitable for coupling to a transmission line, the power is transmitted to the tokamak by a windowless evacuated corrugated waveguide with an overall transmission efficiency of $70 \%-85 \%$. Each waveguide contains a pair of grooved miter bends that can be oriented to polarize the beam to almost any linear or elliptical polarization [48]. The beams from two of the gyrotrons are launched into the tokamak from the low field side using a pair of mirrors with a variable toroidal angular component that allows the user to switch between co and counter injection for maximum experimental flexibility. (This antenna pair was made by the Princeton Plasma Physics Laboratory.) For high density discharges where more than two gyrotrons are required to obtain measurable the ECCD, the beams from the remaining two gyrotrons are launched using a pair of mirrors with a fixed toroidal angular component of $12 \mathrm{deg}$ from radial to drive co current. The poloidal aiming for all the beams can be changed between tokamak pulses. The electron cyclotron waves are absorbed near the intersection of the ray trajectories and the second harmonic of the electron cyclotron frequency. The polarization corresponding to the $\mathrm{X}$-mode dispersion relation is launched in these experiments since it is absorbed strongly for DIII-D conditions. The polarization, propagation, and deposition of the launched electron cyclotron waves have been verified experimentally on DIII-D [49, 50].

A number of diagnostics are required for measuring the ECCD radial profile. The most critical diagnostic is MSE polarimetry using deuterium atoms injected by neutral beams that measures the magnetic field pitch angles at various major radii [51]. The electron density profile is measured using multipulse Thomson scattering [52] along with four $\mathrm{CO}_{2}$ laser interferometers. The electron temperature profile is 
found from a combination of Thomson scattering and electron cyclotron emission (ECE) [53]. Charge exchange recombination (CER) emission of the carbon impurity determines the ion temperature and toroidal rotation profiles [54]; the carbon density profile from CER also determines the effective ion charge $\left(Z_{\text {eff }}\right)$ profile since carbon is the dominant impurity in these plasmas [55]. 


\section{Determination of ECCD Profile From MSE}

Two separate methods have been used on DIII-D to deduce the ECCD profile from MSE polarimetry. In the first method, the non-inductive current drive is determined from the evolution of the poloidal magnetic flux obtained from a magnetic equilibrium reconstruction constrained by the MSE data [56]. However, the localized features in the current profile caused by ECCD are a challenge for this method [57]. In the second method, the measured MSE signals are compared to simulations of the MSE evolution using a model of the rf current drive profile [35]. The parameters of the current drive model — location, width, and magnitude - are adjusted until a best fit between the measured and simulated MSE signals is obtained. Making a comparison between the simulated and measured MSE signals has the advantage that the raw MSE data can be used directly (i.e., the MSE data do not have to be fitted as in an equilibrium reconstruction). In this paper, the ECCD results are obtained using the second method exclusively.

The simulation of the magnetic equilibrium evolution, from which the simulation of the magnetic field pitch angles measured by MSE polarimetry is obtained, is done using the ONETWO code [58] that couples the 1-1/2 D transport calculations to a fixed boundary equilibrium code. This simulation is described in more detail elsewhere [35], but briefly the transport code steps forward in time and evolves the poloidal magnetic field and the parallel electric field $\left(E_{\|}\right)$using Faraday's law and Ohm's law while the parallel current density is determined from Ampère's law. The simulation uses the experimental profiles of the electron density, electron and ion temperatures, toroidal rotation, and effective charge. The flux surface averaged noninductive current densities from NBI and ECCD as well as the bootstrap current are included in Ohm's law in the simulation. The Toray-GA ray tracing code calculates the ECCD profile from the cold plasma dispersion relation and a linear current drive calculation [21, 22, 23]. However, the location, width, and magnitude of the ECCD profile are treated as free parameters to be fitted to the MSE measurement in this analysis.

An example of a co ECCD experiment in an ELMing H-mode plasma is shown in Fig. 1. During the current drive analysis interval, MHD quiescent discharges are required because the plasma current evolution is assumed to be governed by Faraday's law. Therefore, NBI is used during the plasma current ramp up and flat top to raise the electron temperature and slow the resistive evolution of the current profile, thus delaying the onset of sawteeth. The neutral beam also serves as a diagnostic beam for 


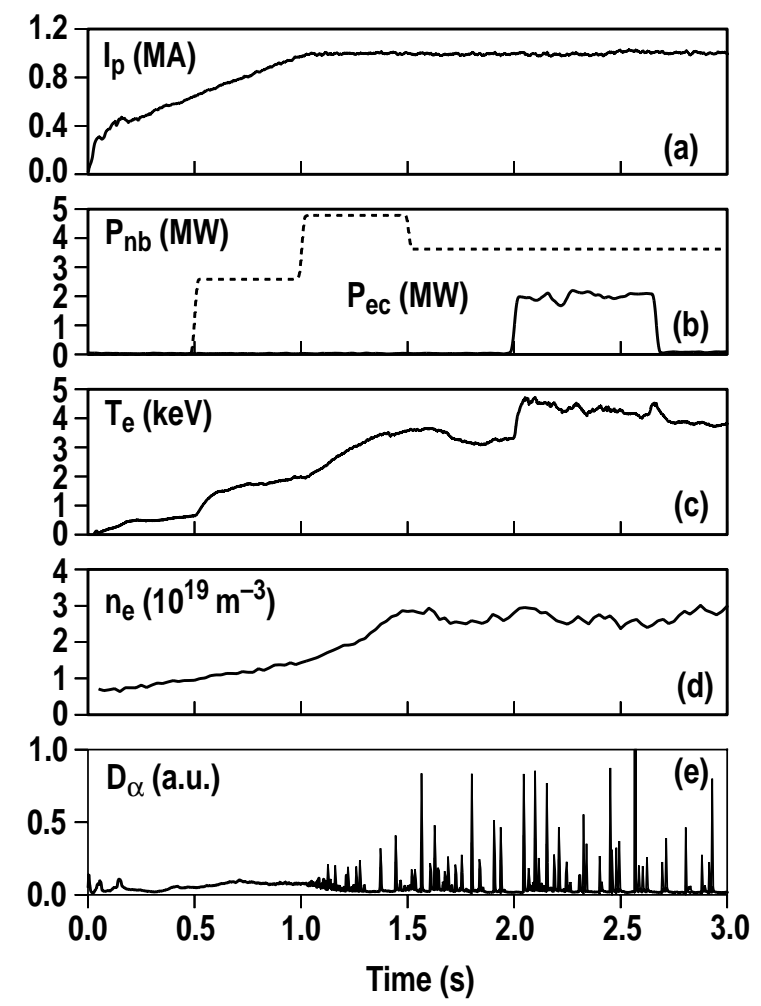

Fig. 1. Time history of ELMing H-mode discharge with co ECCD: (a) plasma current, (b) NBI and ECCD power, (c) central electron temperature from ECE, (d) electron density from line-averaged $\mathrm{CO}_{2}$ laser interferometry, (e) divertor $\mathrm{D}_{\alpha}$ light showing a transition to H-mode after $1 \mathrm{~s}$. 
the CER and MSE polarimetry data. The electron cyclotron waves are injected into the quasistationary phase of the ELMing H-mode plasma before sawtooth activity begins. Since the ECCD deposition location for this case is at a normalized minor radius of $\rho=0.1$, strong central electron heating is observed.

When comparing the measured and simulated MSE signals to determine the ECCD profile, it is convenient to convert the magnetic field pitch angles measured by MSE polarimetry to a quantity that is closely related to the local current density. This can be accomplished using Ampère's law expressed in cylindrical $(R, \phi, z)$ coordinates,

$$
\mu_{0} J_{\phi}=\frac{\partial B_{R}}{\partial z}-\frac{\partial B_{z}}{\partial R},
$$

where $J_{\phi}$ is the toroidal current density, $\mu_{0}$ is the permeability of free space, and $B_{R}$ and $B_{z}$ are the radial and vertical components of the magnetic field. The quantity $B_{z}$ is determined easily from the MSE data by multiplying the measured pitch angle by the vacuum magnetic field $B_{T}$. Although the quantity $B_{R}$ cannot be measured directly by the MSE diagnostic on DIII-D, it is straightforward to show that $B_{R}$ and $B_{z}$ are geometrically related because the magnetic field lines lie in closed surfaces of constant poloidal flux. Modeling the flux surfaces as concentric ellipses yields a relationship between the toroidal current density and vertical magnetic field that is accurate to within $10 \%$ for most plasma shapes of interest [59],

$$
\mu_{0} J_{\phi}=-\frac{B_{z}}{\kappa^{2}\left(R-R_{0}\right)}-\frac{\partial B_{z}}{\partial R},
$$

where $R_{0}$ is major radius of the axis. The solid line in Fig. 2(a) shows the measured change in the toroidal current density profile $\left(\Delta J_{\phi}\right)$ determined using Eq. (3) between the co ECCD case shown in Fig. 1 at $2.25 \mathrm{~s}$ and a similar discharge without ECCD. An example of counter ECCD in similar plasmas is shown in Fig. 2(b). In both cases, the values of $B_{z}$ as a function of $R$ in Eq. (3) come directly from the MSE data. Plotting the differences in $J_{\phi}$ between an ECCD discharge and a no ECCD discharge is favored because systematic uncertainties in the measurements and simulations tend to cancel. In Fig. 2, a large increase (decrease) is observed in the toroidal current density on either side of the magnetic axis at $R_{0}=1.79 \mathrm{~m}$ for co (counter) ECCD.

Given the evolution of the magnetic equilibrium from the ONETWO code simulation discussed at the beginning of this section, it is straightforward to generate time histories of the simulated MSE signals using the viewing geometry of the MSE system for both the ECCD and fiducial (no ECCD) discharges. These simulated profiles of $B_{z}$ as a function of $R$ are then used to generate simulated $\Delta J_{\phi}$ profiles in the same 

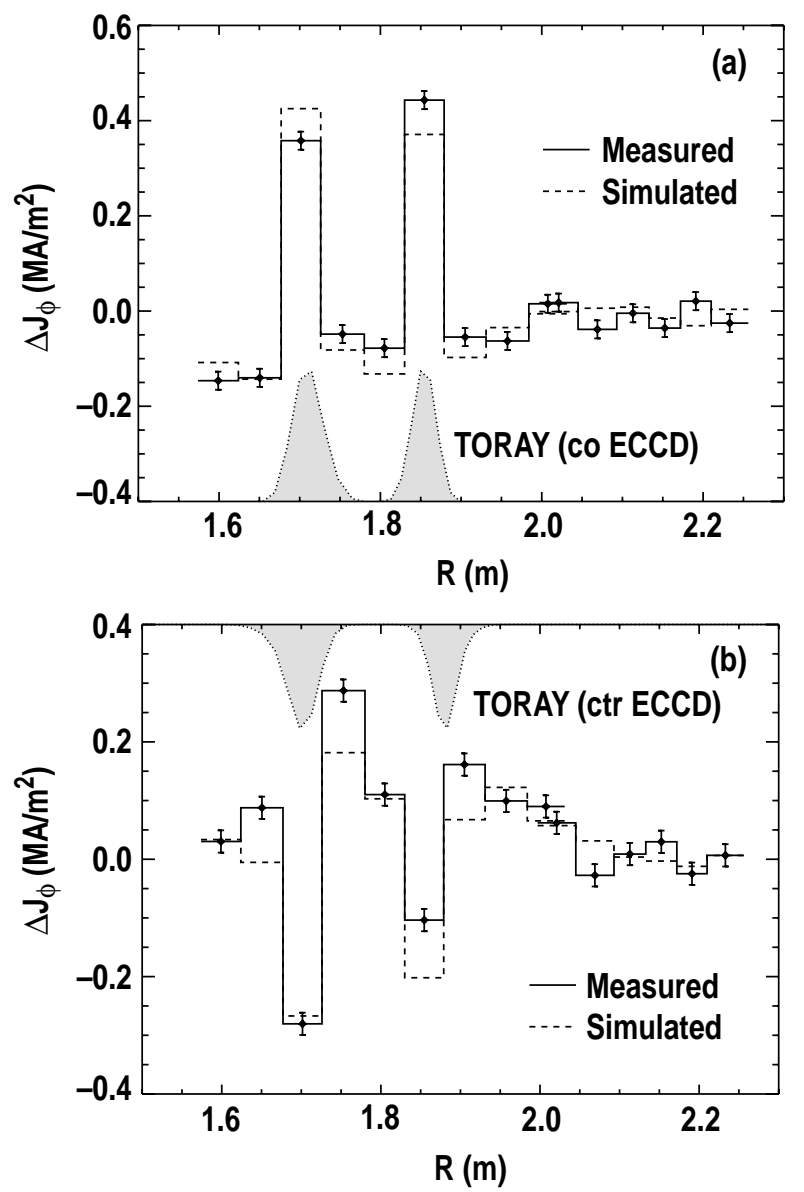

Fig. 2. Change in the measured (solid lines) and simulated (dashed lines) toroidal current density as a function of major radius, determined from Eq. (3), between discharges with and without (a) co ECCD, and (b) counter ECCD. The ECCD profile calculated by the Toray-GA ray tracing code is also indicated (not to scale). 
manner as for the actual MSE data, as shown by the dashed lines in Fig. 2. In addition to the effects of ECCD, these simulations take into account local modifications to $J_{\phi}$ from changes in the ohmic, bootstrap, and neutral beam currents. For Fig. 2(a), the best fit of the simulated MSE signals to the measured MSE signals yields a co ECCD magnitude of $73 \pm 7 \mathrm{kA}$, which is in good agreement with the theoretical value of $75 \mathrm{kA}$ from the CQL3D code, including the effect of $E_{\|}$. For the counter ECCD case in Fig. 2(b), the best fit magnitude is $-58 \pm 9 \mathrm{kA}$, which also is in agreement with the CQL3D value of $-65 \mathrm{kA}$. The location and width of the measured change in $J_{\phi}$ matches the expected ECCD location and width calculated by the Toray-GA ray tracing code. Note that all of the ECCD current lies between two adjacent MSE channels separated by only $0.05 \mathrm{~m}$; thus, there is no experimental indication of ECCD profile broadening caused by radial transport of the energetic electrons (an effect not included in the simulations).

A complicating factor in the ECCD analysis is that the interpretation of the MSE data can be affected by the presence of a large radial electric field $\left(E_{r}\right)[60,61]$. The effect of $E_{r}$ on the MSE signals has not been accounted for in this paper, possibly leading to some systematic uncertainty in the measured ECCD efficiency especially for high beta H-mode plasmas. However, since the ECCD power is much less than the NBI power in these H-mode plasmas, $E_{r}$ is probably similar between the ECCD and fiducial (no ECCD) discharges. In this case, the $E_{r}$ effects on the MSE measurement should tend to cancel when the difference between the toroidal current densities $\left(\Delta J_{\phi}\right)$ is made. Even so, a relatively small change in the $E_{r}$ profile caused by ECCD could explain the asymmetric response (compared to the simulation) of the experimental MSE signals on either side of the plasma axis seen in Fig. 2 [35]. However, Fig. 2 also shows that the best fit simulation does well in averaging out the inboard/outboard asymmetry in the ECCD peaks, and therefore the net effect of $E_{r}$ on the ECCD measurement is probably small for these cases. 


\section{Comparison of Experimental and Theoretical ECCD}

The goal of these ECCD experiments is to validate a predictive model of ECCD. For DIII-D, it is especially important to test the theoretical models of ECCD that are being used to plan future advanced tokamak experiments with current profile control [62]. Since it is known that linear theory does not adequately describe ECCD experiments on DIII-D [31, 32, 35], this paper uses quasilinear Fokker-Planck modeling, including the effects of $E_{\|}$, as calculated by the CQL3D code [24]. The inclusion of $E_{\|}$ in the modeling primarily affects the non-Maxwellian resistivity and leads to only a minor modification of the calculated ECCD. The $E_{\|}$profile used in the CQL3D modeling is determined from a loop voltage profile analysis [56]. Since the electron density and temperature are varied in many of these experiments, it is convenient to normalize out the usual power per particle and collisionality effects shown in Eq. (1) when discussing the current drive efficiency, resulting in a dimensionless ECCD efficiency defined as

$$
\zeta_{e c}=\frac{e^{3}}{\epsilon_{0}^{2}}\left(\frac{I_{e c} R n_{e}}{P_{e c} k T_{e}}\right)=3.27 \frac{I_{e c}(\mathrm{~A}) R(\mathrm{~m}) n_{19}}{P_{e c}(\mathrm{~W}) T_{e}(\mathrm{keV})}
$$

where for simplicity the Coulomb logarithm has been omitted.

In this paper, the main tenets of the CQL3D model of ECCD are examined by determining the current drive dependences as a function of (a) the electron beta $\beta_{e}$, (b) the parallel index of refraction $N_{\|}$, (c) the poloidal deposition location $\theta_{\text {pol }}$, and

(d) the normalized radius of deposition $\rho$. The poloidal angle $\theta_{\text {pol }}$ is defined to be 0 deg on the outboard midplane. The interaction between the electron cyclotron waves and the particles in velocity space are varied in the first two scans, while the interaction in real space is varied in the last two scans. These scans primarily test the effect of electron trapping on the ECCD efficiency. Varying $N_{\|}$also allows the electron trapping effects to be determined for co and counter ECCD separately. Preliminary analysis of some of the data presented in this section has been published previously $[63,64,65]$; a more thorough analysis of the ECCD efficiency from the MSE signals is given here along with an improved determination of the injected ECCD power.

\subsection{Electron Beta Dependence}

The deleterious effects of electron trapping on the ECCD efficiency are expected to decrease at higher electron beta because the relativistic cyclotron resonance shifts away from the trapped-passing boundary in velocity space for low field side launch 
as the electron temperature and/or density rise. This increases the net current drive efficiency toward that of the trapping-free Fisch-Boozer level [66]. This effect is especially important for full current drive scenarios in advanced tokamaks discharges on DIII-D, where ECCD is needed to efficiently drive current at $\rho \approx 0.5$ in high beta discharges [67]. Although the reduction in electron trapping depends upon both absorption and relativistic effects, and therefore is an involved function of $n_{e}$ and $T_{e}$, the experiments reported here are not designed to test these dependences separately. Rather, the local electron beta is used as a convenient figure of merit to represent the expected decrease in trapping effects with increasing density and temperature.

Experiments on the DIII-D tokamak have confirmed the theoretical prediction that the off-axis ECCD efficiency increases with increasing electron beta. The measured increase in the dimensionless ECCD efficiency with increasing local electron beta is shown in Fig. 3 at two radial positions, $\rho=0.3$ and $\rho=0.4$, for electron cyclotron waves with $N_{\|}=0.3$ and deposition above the plasma center $\left(\theta_{\text {pol }}=85 \mathrm{deg}\right)$. The local electron beta is varied by scanning the NBI power and plasma current, and by utilizing both L-mode and H-mode plasmas. The measured $\zeta_{e c}$ is in agreement with the theoretical prediction calculated using the CQL3D code, including the effect of $E_{\|}$, which is seen to increase in Fig. 3 by a factor of 1.5 at $\rho=0.3$ and by a factor of 2.5 at $\rho=0.4$. The increase in $\zeta_{e c}$ with $\beta_{e}$ is stronger at larger $\rho$ because the trapped particle fraction increases with radius. The decrease in $\zeta_{e c}$ with increasing $\rho$, especially apparent in low beta plasmas, is examined in more detail in Section 4.4. The measured increase in $\zeta_{e c}$ with $\beta_{e}$ goes away when the deposition is moved from above the plasma center to the inboard midplane because the effect of trapped electrons on the ECCD efficiency is greatly reduced on the high field side, as discussed in Section 4.3. For example, at $\rho=0.3-0.4$ at $\theta_{\text {pol }}=175 \mathrm{deg}$ near the inboard midplane, the measured ECCD efficiency is $\zeta_{e c}=0.21 \pm 0.02$ at $\beta_{e}=0.2 \%$ and hardly changes to $\zeta_{e c}=0.22 \pm 0.05$ at $\beta_{e}=1.2 \%$, in agreement with the CQL3D calculations.

\section{$4.2 N_{\|}$dependence}

Another method to vary the velocity space interaction between electron cyclotron waves and electrons is to scan the parallel index of refraction. This is done on DIII-D by changing the toroidal injection angle on a shot to shot basis. In most cases, the radius of deposition can be kept fixed by adjusting the poloidal launch angle, although this is ineffective when the deposition is on the inboard midplane $\left(\theta_{p o l}=180 \mathrm{deg}\right)$. Theoretically, the ECCD efficiency is expected to increase with larger $\left|N_{\|}\right|$since the electron cyclotron waves interact with electrons that have higher parallel velocity. 
However, at too high an $\left|N_{\|}\right|$value there are not enough high energy electrons to damp the waves and this effect diminishes.

Figure 4 shows that a scan of the parallel index of refraction from positive to negative values switches the ECCD from the co to the counter direction, with radial injection $\left(N_{\|}=0\right)$ driving little current. The dependence of $\zeta_{e c}$ on $N_{\|}$at the point of absorption is displayed for three off-axis conditions with different poloidal angles of deposition and local electron betas. In Fig. 4, the measured ECCD efficiency is seen to increase with larger $\left|N_{\|}\right|$for both co and counter injection, in agreement with the theoretical value of $\zeta_{e c}$ calculated by the CQL3D code, including the effect of $E_{\|}$. The saturated ECCD efficiency is smaller for the low $\beta_{e}$ cases in Fig. 4(a) and (b) compared to (c), in agreement with the favorable beta scaling of $\zeta_{e c}$ discussed in Section 4.1. In addition, the saturated value of $\zeta_{e c}$ is greater for deposition near the inboard midplane in Fig. 4(b) compared to above the plasma center in Fig. 4(a). This poloidal dependence is examined more fully in Section 4.3. Figure 4 also shows that the counter ECCD tends systematically to be $\approx 10 \%$ less than theoretically expected, a tendency that is not present in the co ECCD data. This systematic deficiency in the counter ECCD is not as large as initially reported [64] because of the larger data set, a more careful analysis of the MSE signals, and a downward refinement of the injected ECCD power.

\subsection{Poloidal dependence}

One of the best ways to investigate the effect of electron trapping on the current drive efficiency is to vary the poloidal location of the ECCD deposition at fixed minor radius. This alters the effect of trapped electrons on the current drive because the local trapped particle fraction varies from small near the inboard midplane $\left(\theta_{\text {pol }}=180 \mathrm{deg}\right)$ to maximum at the outboard midplane $\left(\theta_{p o l}=0 \mathrm{deg}\right)$. Previously on DIII-D, the co ECCD efficiency was measured for a scan of $\theta_{\text {pol }}$ at fixed toroidal injection angle [35]. This somewhat complicated the interpretation of the results since $N_{\|}$at the point of absorption increased as $\theta_{\text {pol }}$ increased. Here, new experiments on DIII-D have varied $\theta_{\text {pol }}$ at fixed $N_{\|}$, as well as fixed $\rho$, by adjusting the toroidal injection angle. Thus, the effects of electron trapping on the current drive efficiency from the poloidal variation can be unambiguously determined.

Figure 5 shows that the measured dimensionless ECCD efficiency increases as the poloidal location of deposition is moved towards the inboard side at fixed minor radius. (The maximum $B_{T}$ of $2.16 \mathrm{~T}$ on DIII-D limits the minimum value of $\theta_{\text {pol }}$ 


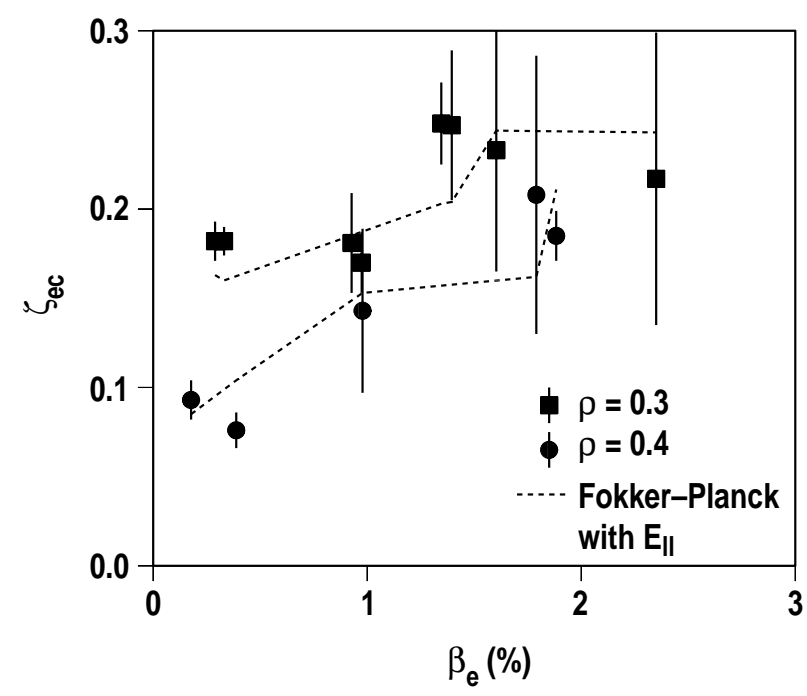

Fig. 3. Measured dependence of the dimensionless ECCD efficiency on the local electron beta for deposition locations of $\rho=0.3$ (squares) and $\rho=0.4$ (circles) with $N_{\|}=0.3$ and $\theta_{\text {pol }}=85 \mathrm{deg}$. The theoretical dependence from the CQL3D code is also shown (dashed lines).
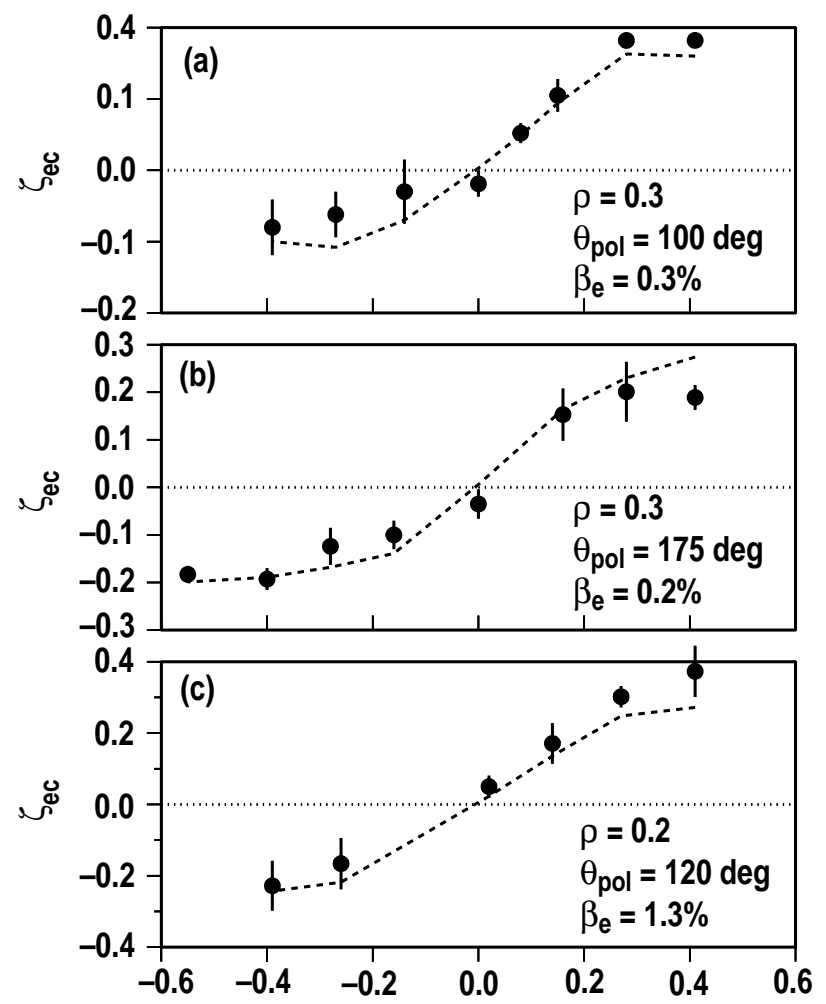

Fig. 4. Experimental dimensionless ECCD efficiency for scans of the parallel index of refraction for two L-mode plasmas [(a) and (b)] and an H-mode plasma [(c)]. The normalized radius and poloidal angle of deposition, and the local electron beta are noted for each scan. The theoretical dependence from the CQL3D code is also shown (dashed lines). 


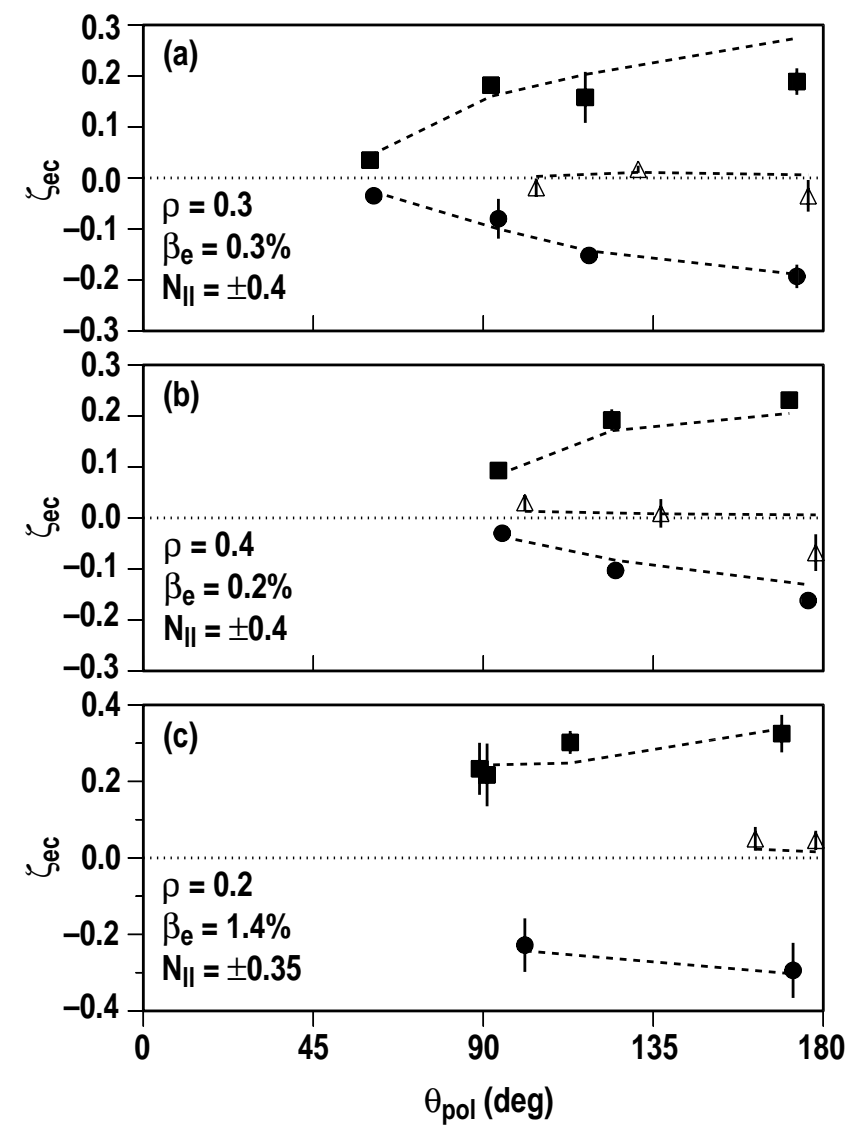

Fig. 5. Experimental dependence of the dimensionless ECCD efficiency on of the poloidal angle of deposition for co (squares) and counter (circles) injection as well as radial injection with $N_{\|}=0$ (triangles). The normalized radius of deposition, local electron beta, and parallel index of refraction for co/counter injection are also noted for each scan. The theoretical dependence calculated by the CQL3D code is also shown (dashed lines). 
to be $\approx 60 \mathrm{deg}$ for off-axis deposition.) This effect is especially apparent in low $\beta_{e}$ plasmas, as seen for the L-mode plasmas in Fig. 5(a) and (b). The weaker dependence in Fig. 5(c) for high $\beta_{e} \mathrm{H}$-mode plasmas is due to the reduced trapping effect at high electron density and temperature, as discussed previously in Section 4.1. In addition, the $\theta_{p o l}$ dependence of $\zeta_{e c}$ is stronger at larger $\rho$, as seen by comparing Fig. 5(a) and (b). This figure also shows that the effect of the trapped electrons on the ECCD efficiency is the same for co and counter injection, while $\zeta_{e c}$ is close to zero for radial injection. The experimental data in Fig. 5 are in agreement with the $\theta_{\text {pol }}$ dependence predicted by the CQL3D code, including the effect of $E_{\|}$, for all cases. Therefore, it is easiest to drive current off-axis when the ECCD location is on the high magnetic field side of the plasma because of reduced electron trapping effects.

\subsection{Radial dependence}

Another effect of electron trapping in tokamaks is that the ECCD efficiency should decrease with increasing minor radius of deposition because the trapped particle fraction increases with increasing inverse aspect ratio [68]. In low beta plasmas, a strong decrease in $\zeta_{e c}$ with increasing radius has been observed previously in co current drive discharges on DIII-D [35] and other devices as mentioned in Section 1 $[11,14,27,28,29,33,34]$. If the decrease in $\zeta_{e c}$ with $\rho$ is due to electron trapping, then the decrease should be less for high $\beta_{e}$ plasmas as well as for deposition locations near the inboard midplane.

Figure 6(a) shows that for low beta L-mode plasmas, the dimensionless ECCD efficiency is measured to decrease rapidly with increasing radius of deposition for both co and counter injection. As seen before, radial injection drives little current. This scan in $\rho$ is done at fixed magnetic field by varying the poloidal steering of the antenna while adjusting the toroidal steering to hold $N_{\|}$constant. The poloidal deposition location in Fig. 6(a) is above the plasma center, where the trapping effects are strong for low beta plasmas as already seen in Section 4.3. The role of electron trapping is confirmed by the radial scan on the inboard midplane in H-mode plasmas shown in Fig. 6(b), where $\zeta_{e c}$ is seen to decrease more slowly with increasing $\rho$. For this scan, the radius of deposition is varied by changing $B_{T}$ while the poloidal steering of the antenna is adjusted to keep the deposition near the inboard midplane. In addition, the toroidal steering of the antenna is adjusted to keep $N_{\|}$fixed. The decrease in $\zeta_{e c}$ with increasing $\rho$ is much less for Fig. 6(b) compared to Fig. 6(a), which is expected since the effects of electron trapping are reduced by increasing the 


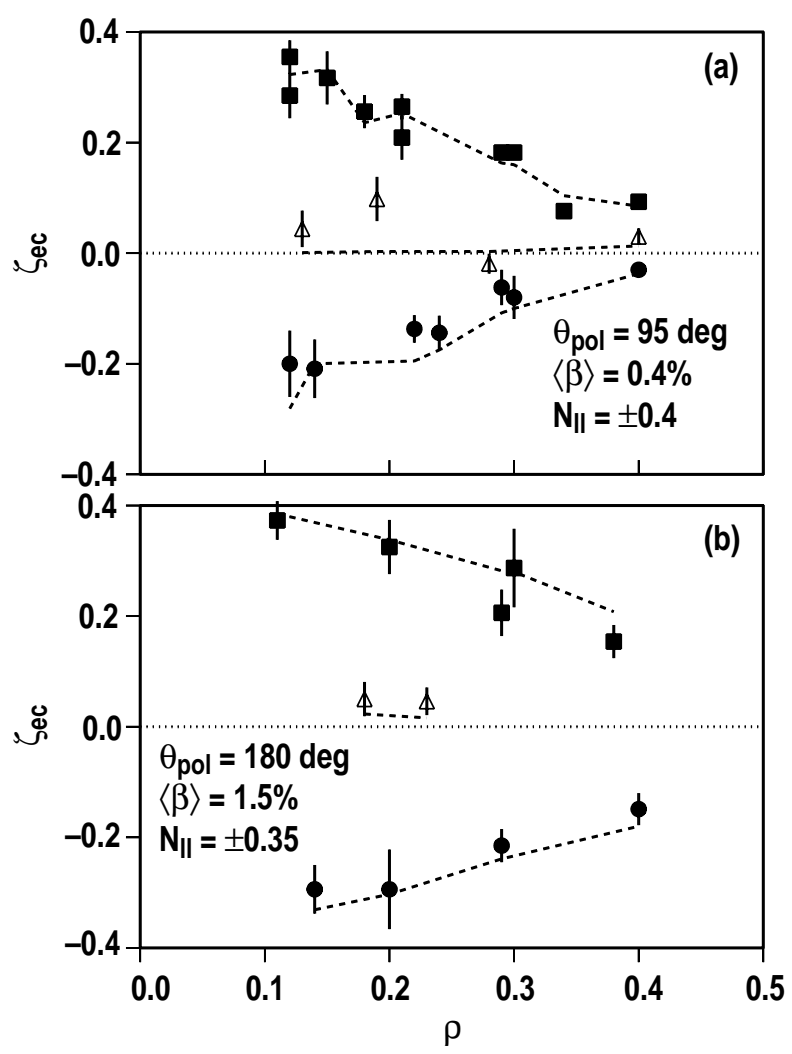

Fig. 6. Measured dimensionless ECCD efficiency for scans of the normalized radius of deposition for co (squares) and counter (circles) injection as well as radial injection with $N_{\|}=0$ (triangles). The poloidal angle of deposition, volume-averaged plasma beta, and parallel index of refraction are also noted for each scan. The theoretical dependence calculated by the CQL3D code is also shown (dashed lines). 
plasma beta and moving the deposition closer to the inboard midplane. In Fig. 6, the theoretical ECCD efficiency from the CQL3D code, including the effect of $E_{\|}$, is seen to be in agreement with the experiment for both the strong trapping and weak trapping situations.

Current profile control with off-axis ECCD is more favorable in high electron beta plasmas since the ECCD efficiency does not decrease much with radius, even for poloidal depositions on the low field side, as seen in Fig. 7. For the poloidal deposition location above the plasma center in Fig. 6(a), the decrease in $\zeta_{e c}$ with $\rho$ extrapolates to nearly zero current drive efficiency at $\rho \approx 0.5$ in these low beta plasmas. However, in advanced tokamak scenarios, the ECCD needs to be located at $\rho \approx 0.5$ for current profile control [58, 62, 67, 69]. Fortunately, Fig. 7 shows that for high beta $\mathrm{H}$-mode plasmas, the measured $\zeta_{e c}$ for co injection decreases little with increasing $\rho$ even for deposition above the plasma center. Replotting the co ECCD data for low beta discharges from Fig. 6(a) in Fig. 7 makes it clear that the detrimental effects of electron trapping on the current drive efficiency are greatly reduced at high electron beta. The CQL3D code predicts that $\zeta_{e c}$ should not decrease much with increasing $\rho$ at high beta, in agreement with the measurements. Thus, in high beta plasmas an ECCD efficiency of $\zeta_{e c} \approx 0.2$ can be expected at $\rho=0.5$ on DIII-D, which is what is required to sustain current profiles with negative central shear for future advanced tokamak scenarios [58, 69].

\subsection{Overview of results}

To summarize the current drive results in this section, the measured ECCD on DIII-D is in good agreement with quasilinear Fokker-Planck calculations from the CQL3D code, including the effect of $E_{\|}$, over a wide range of conditions. This is shown in Fig. 8, where the experimental ECCD (both co and counter) is compared to the CQL3D calculation for the entire DIII-D data set, including scans over a wide range of $\beta_{e}, N_{\|}, \theta_{\text {pol }}$, and $\rho$. The theoretical uncertainties in this figure are due to uncertainties in the launched ECCD power. Figure 8 shows that there is good agreement between theory and experiment for both L-mode and H-mode plasmas, although there is a systematic tendency for the counter ECCD to be $\approx 10 \%$ less than theoretically expected (co ECCD does not show this discrepancy). A statistical comparison between the CQL3D calculations and the ECCD measurements in Fig. 8 yields a reduced $\chi^{2}$ of 1.2 for this data set. The reduced $\chi^{2}$ increases to 5.4 if the linear calculation of the ECCD from the Toray-GA code is used instead since that theory under predicts 
the measured ECCD. Thus, the more complete quasilinear Fokker-Planck theory of ECCD is in better agreement with experiments than the linear theory [35].

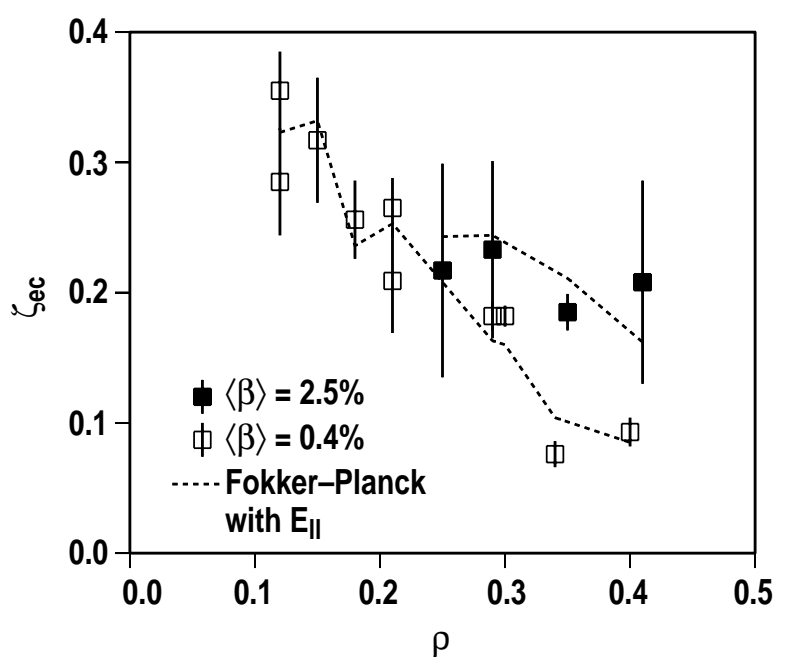

Fig. 7. Measured dependence of the dimensionless ECCD efficiency on the normalized radius of deposition for low beta L-mode plasmas with $\theta_{\text {pol }}=95 \mathrm{deg}$ and $N_{\|}=0.4$ (open squares) and high beta H-mode plasmas with $\theta_{\text {pol }}=90 \mathrm{deg}$ and $N_{\|}=0.3$ (filled squares). The theoretical dependence for both conditions from the CQL3D code is also shown (dashed lines).

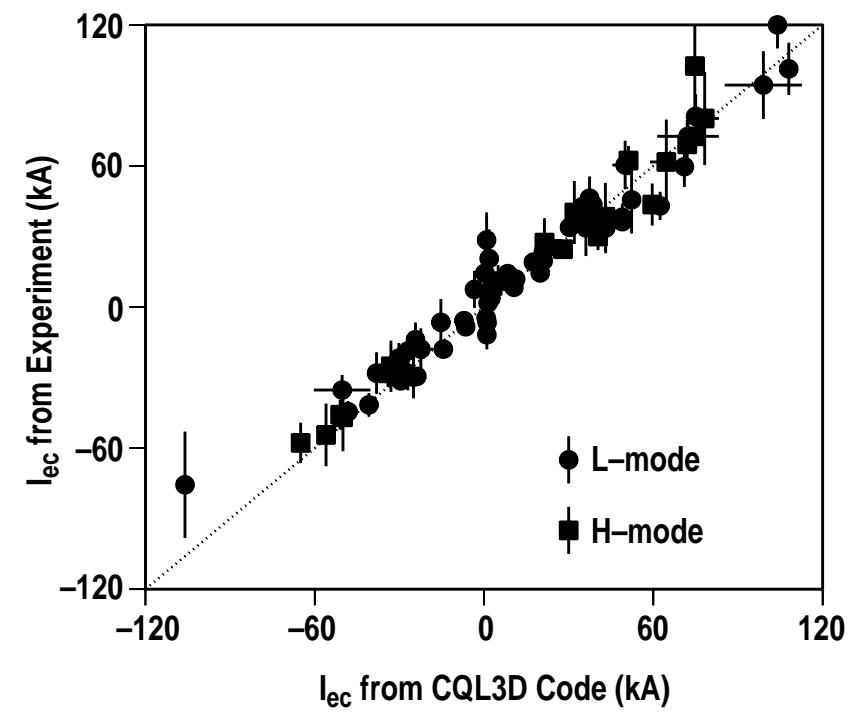

Fig. 8. Comparison of measured and theoretical ECCD over a range of parameters for both L-mode (circles) and H-mode (squares) plasmas. The theoretical ECCD is calculated using a quasilinear Fokker-Planck code including the effect of $E_{\|}$. 


\section{Conclusions}

The physics basis for localized, off-axis current drive has been solidified by recent ECCD experiments on the DIII-D tokamak. Clear evidence for localized current drive using electron cyclotron waves is seen from changes in the magnetic field pitch angles measured by MSE polarimetry. The width of the current drive profile is in agreement with the expected width from ray tracing calculations, indication that radial transport of the current carrying electrons is small. Scans of the parallel index of refraction show that both co and counter ECCD are clearly observed, while radial injection is found to drive little current. The measured ECCD is in good agreement with quasilinear Fokker-Planck calculations, including the effect of the parallel electric field, over a wide range of parameters in these experiments, although the counter ECCD tends systematically to be $\approx 10 \%$ less than theoretrically expected (co ECCD does not show this discrepancy).

These experiments have examined the effect of electron trapping on the dimensionless ECCD efficiency by varying independently the radial and poloidal deposition locations as well as the local electron beta for both co and counter injection. For each scan, the parallel index of refraction is kept nearly fixed. The ECCD efficiency is found to increase with increasing local electron beta, especially for off-axis absorption. This can be explained theoretically by the stronger damping of the electron cyclotron waves with higher density and temperature as well as relativistic effects, causing the resonant interaction to move away from the trapped-passing boundary which reduces the deleterious effects of electron trapping and improves the ECCD efficiency. The detrimental effects of electron trapping are also readily seen in the decrease in the measured ECCD efficiency for co and counter injection as the deposition is moved off-axis and towards the high field side, especially for low beta plasmas. For high beta plasmas, these dependencies are greatly reduced since electron trapping is less important. The measured ECCD efficiency for off-axis deposition in high beta H-mode plasmas on DIII-D is equal to the value needed for future advanced tokamak scenarios that will use ECCD for current profile control. Thus, the DIII-D tokamak is making excellent progress towards experimentally validating a predictive model of ECCD that can be used to plan future applications of current drive for MHD stabilization and current profile control. 


\section{References}

${ }^{(a)}$ Princeton University, Princeton, New Jersey.

${ }^{(b)}$ CompX, Del Mar, California.

(c) Lehigh University, Bethlehem, Pennsylvania.

(d) Lawrence Livermore National Laboratory, Livermore, California.

[1] Gantenbein, G., et al., Phys. Rev. Lett. 85 (2000) 1242.

[2] Zohm, H., et al., Phys. Plasmas 8 (2001) 2009.

[3] Isayama, A., et al., Plasma Phys. Control. Fusion 42 (2000) L37.

[4] Prater, R., et al., in Fusion Energy 2000 (Proc. 18th Int. Conf. Sorrento, 2000), IAEA, Vienna (2001) EX8/1.

[5] La Haye, R.J., et al., "Control of Neoclassical Tearing Modes in DIII-D," to be published in Phys. Plasmas.

[6] Alikaev, V.V., et al., Plasma Phys. Rep. 26 (2000) 177.

[7] Razumova, K.A., et al., Plasma Phys. Control. Fusion 42 (2000) 973.

[8] Ryter, F. et al., in Strong Microwaves in Plasmas (Proc. 4th Int. Conf. Nizhny Novgorod, 1999), Vol. 1, Russian Academy of Sciences, Nizhny Novgorod (2000) 134.

[9] Kislov, D.A., et al., in Controlled Fusion and Plasma Physics (Proc. 22nd Eur. Conf. Bournemouth, 1995), Vol. 19C, Part I, European Physical Society, Geneva (1995) 369 .

[10] Asakawa, M., Tanaka, H., Maekawa, T., Terumichi, Y., Fusion Eng and Design 53 (2001) 237.

[11] Sauter, O., et al., Phys Plasmas 8 (2001) 2199.

[12] Ando, A., et al., Phys. Rev. Lett. 56 (1986) 2180.

[13] Tanaka, H., et al., Nucl. Fusion 31 (1991) 1673.

[14] Sauter, O., et al., Phys. Rev. Lett. 84 (2000) 3322. 
[15] Erckmann, V., Gasparino, U., Maaßberg, H., Plasma Phys. Control. Fusion 34 (1992) 1917.

[16] Fisch, N.J., Boozer, A.H., Phys. Rev. Lett. 45 (1980) 720.

[17] Fisch, N.J., Rev. Mod. Phys. 59 (1987) 175.

[18] Ohkawa, T., "Steady-State Operation of Tokamaks by R-F Heating," General Atomics Report GA-A13847 (1976).

[19] Ehst, D.A., Karney, C.F.F., Nucl. Fusion 31 (1991) 1933.

[20] Harvey, R.W., McCoy, M.G., Kerbel, G.D., Phys. Rev. Lett. 62 (1989) 426.

[21] Cohen, R.H., Phys. Fluids 30 (1987) 2442.

[22] Matsuda, K., IEEE Trans. Plasma Sci. 17 (1989) 6.

[23] Lin-Liu, Y.R., et al., in Controlled Fusion and Plasma Physics (Proc. 26th Eur. Conf. Maastricht, 1999), Vol. 23J, European Physical Society, Geneva (1999) 1245.

[24] Harvey, R.W., McCoy, M.G., in Proceedings of the IAEA Technical Committee Meeting, Montreal, 1992 (IAEA, Vienna, 1993) 498.

[25] Erckmann, V., Gasparino, V., Plasma Phys. Control. Fusion 36 (1994) 1869.

[26] Lloyd, Brian, Plasma Phys. Control. Fusion 40 (1998) A119.

[27] Razumova, K.A., et al., Phys. Plasmas 1 (1994) 1554.

[28] Alikaev, V.V., et al., Nucl. Fusion 35 (1995) 369.

[29] Esipchuk, Yu. V, Plasma Phys. Control. Fusion 37 (1995) A267.

[30] Todd, T.N., et al., Plasma Phys. Control. Fusion 35 (1993) B231.

[31] James, R.A., et al., Phys. Rev. A 45 (1992) 8783.

[32] Petty, C.C., et al., Nucl. Fusion 35 (1995) 773.

[33] Schüller, F.C., Polman, R.W., in Strong Microwaves in Plasmas (Proc. 4th Int. Conf. Nizhny Novgorod, 1999), Vol. 1, Russian Academy of Sciences, Nizhny Novgorod (2000) 65.

[34] Westerhof, E., et al., Fusion Eng. and Design 53 (2001) 259. 
[35] Petty, C.C., et al., Nucl. Fusion 41 (2001) 551.

[36] Erckmann, V., et al., in Fusion Energy 1998 (Proc. 17th Int. Conf. Yokohama, 1998), Vol. 2, IAEA, Vienna (1999) 639.

[37] Geiger, J., et al., in Fusion Energy 2000 (Proc. 18th Int. Conf. Sorrento, 2000), IAEA, Vienna (2001) EXP4/04.

[38] Erckmann, V., et al., Fusion Eng. and Design 53 (2001) 365.

[39] Luce, T.C., et al., Phys. Rev. Lett. 83 (1999) 4550.

[40] Luce, T.C., et al., Plasma Phys. Control. Fusion 411 (1999) B119.

[41] Luce, T.C., Petty, C.C., Schuster, D.I., Makowski, M.A., Fusion Eng. and Design 53 (2001) 337.

[42] Isayama, A. et al., in Radiofrequency Power in Plasmas (Proc. 14th Int. Conf. Oxnard, 2001), AIP, New York (2001) 267.

[43] Suzuki, T., et al., Plasma Phys. Control. Fusion 44 (2002) 1.

[44] Luxon, J.L., Davis, L.G., Fusion Technol. 8 (1985) 441.

[45] Callis, R.W., et al., in Fusion Technol. (Proc. 20th Symp. Marseille, 1998), Vol. 1, Association EURATOM-CEA, Saint-Paul-Lez-Durance (1998) 315.

[46] Lohr, J., et al., in Infrared and Millimeter Waves (Proc. 23rd Int. Conf. Colchester, 1998), University of Essex, Colchester (1998) 269.

[47] Lohr, J., et al., in Radiofrequency Power in Plasmas (Proc. 14th Int. Conf. Oxnard, 2001), AIP, New York (2001) 314.

[48] Doane, J., Int. J. Infrared Millimeter Waves 13 (1992) 1727.

[49] Petty, C.C., et al., in Radiofrequency Power in Plasmas (Proc. 13th Int. Conf. Annapolis, 1999), AIP, New York (1999) 245.

[50] Petty, C.C., et al., in Strong Microwaves in Plasmas (Proc. 4th Int. Conf. Nizhny Novgorod, 1999), Vol. 1, Russian Academy of Sciences, Nizhny Novgorod (2000) 41.

[51] Rice, B.W., Burrell, K.H., Lao, L.L., Lin-Liu, Y.R., Phys. Rev. Lett. 79 (1997) 2694. 
[52] Carlstrom, T.N., Campbell, G.L., Deboo, J.C., Evanko, R., Evans, J., Rev. Sci. Instrum. 63 (1992) 4901.

[53] Wang, Z., et al., in Electron Cyclotron Emission and Electron Cyclotron Heating (Proc. 9th Int. Workshop Borrego Springs, 1995), World Scientific, Singapore (1995) 427.

[54] Gohil, P., Burrell, K.H., Groebner, R.J., Seraydarian, R.P., Rev. Sci. Instrum. 61 (1990) 2949.

[55] Wade, M.R., et al., Phys. Plasmas 2 (1995) 2357.

[56] Forest, C.B., et al., Phys. Rev. Lett. 73 (1994) 2444.

[57] Lao, L.L., et al., in Radiofrequency Power in Plasmas (Proc. 14th Int. Conf. Oxnard, 2001), AIP, New York (2001) 310.

[58] St. John, H., Taylor, T.S., Lin-Liu, Y.R., Turnbull, A.D., in Plasma Physics and Controlled Nuclear Fusion Research 1994 (Proc. 15th Int. Conf. Seville, 1994), Vol. 3, IAEA, Vienna (1996) 603.

[59] Petty, C.C., Fox, W.R., Luce, T.C., Makowski, M.A., Suzuki, T., "Analysis of Current Drive Using MSE Polarimetry Without Equilibrium Reconstruction," submitted to Nucl. Fusion.

[60] Zarnstorff, M.C., Levinton, F.M., Batha, S.H., Synakowski, E.J., Phys. Plasmas 4 (1997) 1097.

[61] Rice, B.W., Burrell, K.H., Lao, L.L., Nucl. Fusion 37 (1997) 517.

[62] Rice, B.W., et al., Nucl. Fusion 39 (1999) 1855.

[63] Petty, C.C., et al., in Radiofrequency Power in Plasmas (Proc. 14th Int. Conf. Oxnard, 2001), AIP, New York (2001) 275.

[64] Petty, C.C., et al., in Controlled Fusion and Plasma Physics (Proc. 28th Eur. Conf. Funchal, 2001), Vol. 25A, European Physical Society, Geneva (2001) 1161.

[65] Kirneva, N.A., Plasma Phys. Control. Fusion 43 (2001) A195.

[66] Prater, R., Harvey, R.W., Lin-Liu, Y.R., Luce, T.C., Petty, C.C., in Radiofrequency Power in Plasmas (Proc. 14th Int. Conf. Oxnard, 2001), AIP, New York (2001) 302. 
[67] Petty, C.C., et al., Plasma Phys. Control. Fusion 42, B75.

[68] Lin-Liu, Y.R., Miller, R.L., Phys. Plasmas 2 (1995) 1666.

[69] Turnbull, A.D., Taylor, T.S., Lin-Liu, Y.R., St. John, H., Phys. Rev. Lett. 74 (1995) 718. 


\section{Acknowledgments}

This work is supported by the U.S. Department of Energy under Contract Nos. DE-AC03-99ER54463 and W-7405-ENG-48, and Grant Nos. DE-FG03-99ER54141 and DE-FG02-92ER54141. One of us (W.R. Fox) is funded by the National Undergraduate Fellowships in Plasma Physics and Fusion Energy Sciences from the USDOE.

The authors would like to thank Y. Gorelov for determining the ECCD powers used in this paper. 